TITLE:

\title{
DYNAMICAL SYSTEMS ON DRAGON DOMAINS
}

$\operatorname{AUTHOR}(\mathrm{S})$ :

MIZUTANI, Masahiro; ITO, Shunji

CITATION:

MIZUTANI, Masahiro ...[et al]. DYNAMICAL SYSTEMS ON DRAGON

DOMAINS. 数理解析研究所講究録 1985, 574: 144-165

ISSUE DATE:

1985-12

URL:

http://hdl.handle.net/2433/99209

RIGHT: 
DYNAMICAL SYSTEMS ON DRAGON DOMAINS

早稲田大学理工.応物 水谷正大 (Masahiro MIZUTANI)

津田塾大学.数 学 伊藤俊次（Shunji ITO）

\section{ABSTRACT}

Dynamical systems on fractal domains are studied. These domains are called twindragon. tetradragon and cross dragon respectively.

\section{INTRODUCTION}

we can see the following fact in knuth ${ }^{1}$ : For any complex number there exists the zero-one sequence $a_{k} \cdot a_{k-1}, \ldots a_{0}, a_{-1}, \ldots$ such that

$$
z=\Sigma_{-\infty} \leqq j \leqq k^{a}(i-1)^{j},
$$

that is, eury complex number has a "binary" representation with base i-1. This fact suggests an existence of a number theoretic dynamical system $\left(\hat{\mathrm{X}}_{\mathrm{i}-1}, \hat{\mathrm{T}}_{\mathrm{i}-1}, \hat{\mu}\right)$ which induces the binary expansion. Actually if there exists a domain $\hat{X}_{1-1}$ and its partition $\left\{\hat{X}_{i-1,0}, \hat{x}_{i-1,1}\right\}$ such that 
(i) $\hat{x}_{i-1}=\hat{x}_{i-1,0} \cup \hat{x}_{i-1,1}$ and int $\left(\hat{x}_{i-1,0}\right) \cap \operatorname{int}\left(\hat{x}_{i-1,1}\right)=\phi$

(ii) $\hat{\mathrm{x}}_{\mathrm{i}-1}=(\mathrm{i}-1) \hat{\mathrm{x}}_{\mathrm{i}-1,0}=(\mathrm{i}-1) \hat{\mathrm{x}}_{\mathrm{i}-1,1}-1$,

then the transformation $\hat{T}_{i-1}$ on $\hat{x}_{i-1}$ such that

$$
\hat{\mathrm{T}}_{i-1} z=(i-1) z-\hat{a}((i-1) z)
$$

where $\hat{a}(z)=j$ if $z \in j+\hat{x}_{i-1, j}, j=0,1$, induces the binary expansion.

On the other hand we can see also the followings in Davis and $K_{n u t h}{ }^{2)}$ : for any complex integer $m+i n$. there exists a revolving sequence of finite length $\delta_{1}, \delta_{2}, \ldots \delta_{k}$ such that

$$
m+i n=\sum_{j=1}^{k} \delta_{j}(1+i)^{k-j}
$$

where the revolving sequence $\left(\delta_{1}, \delta_{2}, \ldots\right)$ is defined by the following conditions:

(i) $\delta_{j} \in\{0,1,-i,-1, i\}$

(ii) if $\left(\delta_{1}, \ldots \delta_{j}\right) \neq(0, \ldots, 0)$

then $\delta_{j+1}=0$ or $(-i) \delta_{k_{0}}$ for all $j \in \mathbb{N}$

where $k_{0}=\max \left\{k ; \delta_{k} \neq 0,1 \leqq k \leqq j\right\}$

(i i i) if $\left(\delta_{1}, \ldots, \delta_{j}\right)=(0, \ldots, 0)$

then $\delta_{j+1} \in\{0, \pm 1, \pm i\}$.

This fact also suggests an existence of a number theoretic dynamical system $(X, T, V)$ which induces the revolving expansion

$$
z=\sum_{k=1}^{\infty} \delta_{k}(1+i)^{-k}
$$

We consider the existence problem of above dynamical systems $\left(\hat{x}_{1-i} \cdot \hat{T}_{1-i}, \hat{\mu}\right)$ and $(X, T, \nu)$ and show that the boundaries of these domains $\hat{x}_{i-1}$ and $x$. called the twindragon 
and the tetradragon respectively, are indeed fractal sets ${ }^{3}$ ). Moreover we propose a new construction of the dragon different from the paper folding process and consider a dynamical system $(Y, S, \lambda)$ on a domain tiled by four dragon which is not the tetradragon. called a cross dragon. Suprisingly we can show that this cross dragon system ${ }^{4}$ is actually the dual system for a very simple group endomorphism $\mathrm{T}_{\mathrm{L}}$ on $\mathrm{T}^{2}$ such that

$$
T_{L}\left(\begin{array}{l}
x \\
y
\end{array}\right)=\left(\begin{array}{rr}
1 & -1 \\
1 & 1
\end{array}\right)\left(\begin{array}{l}
x \\
y
\end{array}\right)-\left(\begin{array}{l}
{[x-y]} \\
{[x+y]}
\end{array}\right) \text {. }
$$

2. BINARY EXPANSION ON TUINDRAGON

Firstly consider the binary expansion with base $(1+i)$;

$$
z=\sum_{k=1}^{\infty} \varepsilon_{k}(1+i)^{-k} \text {, }
$$

where $\varepsilon_{k} \in\{0 . i\}$ for all $k \in \mathbb{N}$. If there exist a dynamical system $\left(X_{1+i} \cdot T_{1+i}, \mu\right)$ which induces this representation, then the domain must be the limit points of $Q_{n}$ such that

$$
Q_{n}=\left\{\Sigma_{k=1}^{n} \varepsilon_{k}(1+i)^{-k} ; \varepsilon_{k} \in\{0, i\}\right\}
$$

and also $X_{1+i, \varepsilon}, \varepsilon=0, i$, must be the limit point of $Q_{n, \varepsilon}=$ $\left\{\sum_{k=1}^{n} \varepsilon_{k}(1+i)^{-k}: \varepsilon_{1}=\varepsilon\right\}$ in the Hausdorf $f$ metric space $(g, d)$. For after discussions we put

$$
P_{n+1}=(1+i) Q_{n} \quad \text { for } n \geqq 1 .
$$

that is.

$$
P_{n+1}=\left\{\sum_{k=0}^{n-1} \varepsilon_{k}(1+i)^{-k}: \varepsilon_{k} \in\{0 . i\}\right\} .
$$


We consider the shape and properties of $x_{1+i}$ such that $\mathrm{d}\left(\mathrm{X}_{1+\mathrm{i}}, \mathrm{P}_{\mathrm{n}}\right) \rightarrow 0$ as $\mathrm{n} \rightarrow \infty$. Let $\mathrm{U}$ be a closed square with vertices $0,1,1-i$ and $-i$, and for each point $x\left(\varepsilon_{0}, \ldots \varepsilon_{n-1}\right) \in P_{n+1}$ we prepare the neighborhood of a point $x\left(\varepsilon_{0} \ldots \varepsilon_{n-1}\right)$ such that

$$
\left.U_{x\left(\varepsilon_{0}\right.} \ldots \varepsilon_{n-1}\right)=x\left(\varepsilon_{0} \ldots \ldots \varepsilon_{n-1}\right)+(1+i)^{-(n-1)} U \text {, }
$$

and let $F_{n+1}$ and $B_{n+1}$ be

and

$$
\left.F_{n+1}=U_{x \in P_{n+1}} U_{x\left(\varepsilon_{0}\right.} \ldots \varepsilon_{n-1}\right)
$$

$$
B_{n+1}=\partial F_{n+1}
$$

respectively. We call $B_{n+1}$ a $(n+1)-$ step Bernoulli boundary (Fig.1(a)). We give the names for each side of $B_{n+1}$ as a following way: For each $n \geqq 1$ we name each side of the square $(1+i)^{-(n-1)} U$ A, $B, A^{-1}$ and $B^{-1}$ respectively, then we obtain names of each side of the neighborhood of point $x\left(\varepsilon_{0} \ldots \varepsilon_{n-1}\right)$ according to above namings. Therefore we can read a sequence of names for $B_{n+1}$ as to be $A_{n+1,1}, A_{n+1,2}, \cdots, A_{n+1, m(n)}$ where $A_{n+1,1}$ is a first name of a side $\left[0,(1+i)^{-(n-1)}(-i)\right]$ and $A_{n+1, k} \in\left\{A, A^{-1}, B, B^{-1}\right\}$ is a name of $k-t h$ side of $B_{n+1}$.

\section{$\operatorname{Lemma(2.1)}$}

The names of each side of $B_{n+1}$ are obtained from these of $B_{n}$ by the substitution $\Theta: A \rightarrow A B, B \rightarrow A^{-1} B$, that is. the names of each side of $B_{n+1}=\Theta^{n}\left(A B A^{-1} B^{-1}\right)$.

By the way, recall the notation by Dekking ${ }^{5), 6)}$ for our purpose. Let $G$ be a finite set of symbols, $G^{*}$ the free 


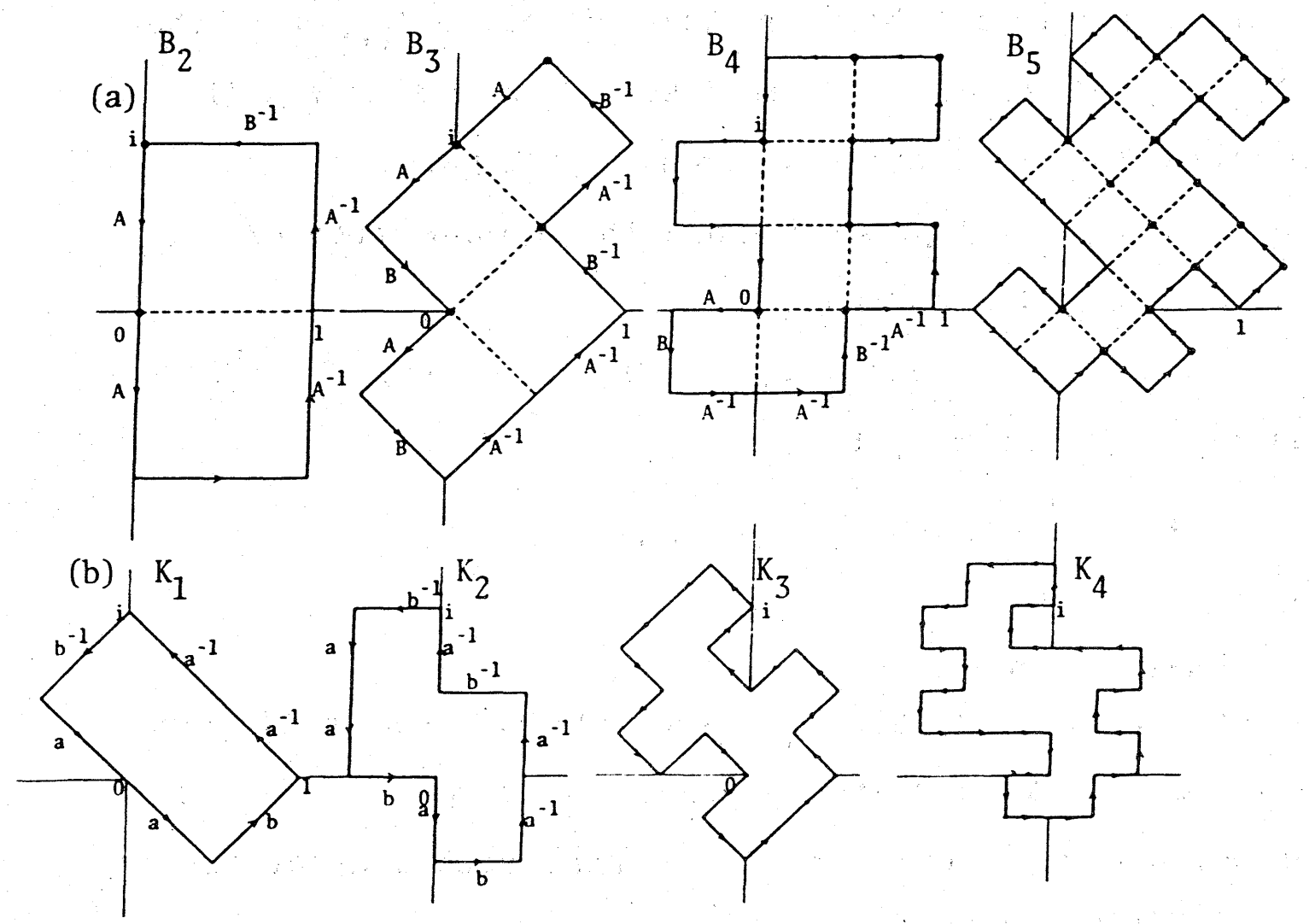

Fig. 1: Bernoul1i boundary $B_{n}$ and Dragon boundary $K_{n-1}$.

semigroup generated by $G$ and $\theta: G^{*} \rightarrow G^{*}$ a semigroup endomorphism. Let $\mathrm{f}: \mathrm{G}^{*} \rightarrow \mathbb{C}$ be a homeomorphism which satisfies $f(v w)=f(v)+f(w) \cdot f\left(v^{-1}\right)=-f(v)$

for all words $V, W \in G^{*}$. Define a map $K: S^{*} \rightarrow \mathbb{C}$. which satisfies $K[V W]=K[V] \cup(K[W]+f(V))$

for all reduced words $V, w \in G^{*}$, by $K[s]=\{t f(s) ; 0 \leqq t \leqq 1\}$ for $s \in G$.

This makes $K\left[s_{1} \ldots s_{m}\right]$ the polygonal line with vertices at $0 . f\left(s_{1}\right) \cdot f\left(s_{1}\right)+f\left(s_{2}\right), \ldots . f\left(s_{1}\right)+\ldots+f\left(s_{m}\right)$ 
Especially we consider here a following case,

$$
G=\{a, b\}, f(a)=1, f(b)=i \text {, }
$$

and

$$
\theta: \quad \theta(a)=a b, \quad \theta(b)=a^{-1} b .
$$

Then the following relation holds

$$
f \theta=(1+i) f \text {. }
$$

we put

$$
K_{n}=(1+i)^{-n_{k}} K\left[\theta^{n}\left(a b a^{-1} b^{-1}\right)\right],
$$

and call $\mathrm{K}_{\mathrm{n}}$ a n-step dragon boundary (Fig.l(b)).

Theorem (Dekking ${ }^{5), 6)}$ )

(1) There exists a closed curve $k_{\theta}$ such that

$$
(1+i)^{-n_{K}\left[\theta^{n}\left(a b a^{-1} b^{-1}\right)\right] \rightarrow K_{\theta}} \text { as } n \rightarrow \infty
$$

in the Hausdorf $f$ metric,

(2) $\operatorname{dim} \mathrm{H}_{\theta}=2 \log \beta_{0} / \log 2$, where $\beta_{0}$ is a unique real root of $\beta^{3}-\beta^{2}-2=0$.

$\mathrm{K}_{\theta}$ is called a dragon boundary or a twindragon skin because of lemma(3.2).

We obtain a following relation between $B_{n}$ and $k_{n}$.

\section{Lemma (2.2)}

$$
B_{n+1}=2(1+i)^{-1}\left(K_{n-1}\right) \text {. }
$$

\section{Corollary(2.3)}

Let $X_{1+i}$ and $x_{1+i, \varepsilon}, \varepsilon=0, i$, be convergent sets of $Q_{n}$ and $Q_{n, \varepsilon}(\varepsilon=0, i)$ in the Hausdorff metric (Fig.2), then 
150

(1) $\partial x_{1+i}$ is similar to the dragon boundary.

(2) $x_{1+i}=x_{1+i, 0} \cup x_{1+i . i}$.

(3) $\quad x_{1+i}=(1+i) x_{1+i, 0}=(1+i) x_{1+i . i}-i$.

(4) $\operatorname{dim}_{H}\left(x_{1+i .0} \cap x_{1+i, i}\right)=2 \log \beta_{0} / \log 2$.

Thus we can define a transformation $T_{1+i}$ on $x_{1+i}$ by

$$
T_{1+i} z=(1+i) z-[(1+i) z]_{1+i}
$$

where a digit $\left.{ }^{[z]}\right]_{1+i}$ be

$$
[w]_{1+i}=\left\{\begin{array}{ll}
0 & \text { if } w \in X_{1+i} .0 \\
i & \text { if } w \in i+X_{1+i} . i
\end{array}\right. \text {. }
$$

Then we obtain

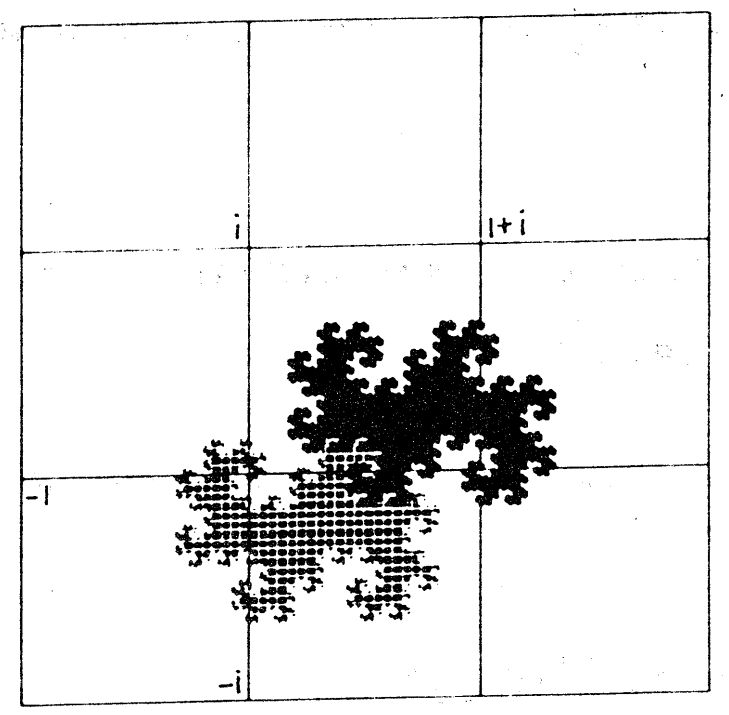

Fig. 2: Domain $\mathrm{X}_{1+\mathrm{i}}$.

7 


\section{Theorem(2.1)}

(1) The transformation $\left(X_{1+i}, T_{1+i}\right)$ induces the complex binary expansion for a.e. $z \in X_{1+i}$ such that

$$
z=\sum_{k=1}^{\infty} a_{k}(z)(1+i)^{-k}
$$

where $a_{k}(z)=\left[(1+i) T_{1+i} k-1 z\right]_{1+i}$.

(2) The Lebesgue measure $\mu$ is invariant with respect to $\left(\mathrm{X}_{1+\mathrm{i}}, \mathrm{T}_{1+\mathrm{i}}\right)$ and the dynamical system $\left(\mathrm{X}_{1+\mathrm{i}}, \mathrm{T}_{1+\mathrm{i}} \cdot \mu\right)$ is isomorphic to the two states Bernoulli system.

\section{Remark:}

(i) Put

$$
x_{1-i}=\overline{x_{1+i}} \cdot \quad[w]_{1-i}=\overline{[w]_{1+i}} \text {. }
$$

where - means to take a complex complex conjugate, and

$$
T_{1-i} z=(1-i) z-[(1-i) z]_{1-i} \text { for } z \in X_{1-i} \text {. }
$$

Then dynamical system $\left(\mathrm{X}_{1-\mathrm{i}} \cdot \mathrm{T}_{1-\mathrm{i}} \cdot \mu\right)$ induces the complex binary expansion with base $(1-i)$.

(i i) Putting

$$
\begin{aligned}
& x_{i-1}=\frac{1-2 i}{5}+x_{1-i} \\
& x_{i-1, \varepsilon}=\frac{1-2 i}{5}+x_{1-i, \varepsilon}, \varepsilon=0,-i .
\end{aligned}
$$

and

$$
T_{i-1} z=(i-1) z-[(i-1) z]_{i-1} .
$$

where $[w]_{i-1}=\varepsilon$ if $w \in \varepsilon+X_{i-1}$, then $\left(X_{i-1}, T_{i-1}, \mu\right)$ is well defined and induces the complex binary expansion with base $(i-1)$.

(i i i) Taking a complex conjugate of $\left(\mathrm{X}_{\mathrm{i}-1}, \mathrm{~T}_{\mathrm{i}-1}, \mu\right)$, then the dynamical system $\left(x_{-1-i}, T_{-1-i}, \mu\right)$ is obtained and induces the 
complex binary expansion with base $(-1-i)$.

We remark that the sets $x_{i-1}$ and $x_{-1-i}$ include the origin as an internal point respectively.

(iv) The set of the twin dragons $\left\{X_{1+i}+m+i n ; m+i n \in Z(i)\right\}$ tiles the whole plane, that is.

$$
U_{m+i n} X_{1+i}+m+i n=\mathbb{C}
$$

and

$$
\mu\left(U_{m+i n} \partial\left(x_{1+i}+m+i n\right)\right)=0
$$

3. REVOLVING EXPANSION ON TETRADRAGON

Let $M=\left(M_{j, k}\right), j, k \in\{0,1,2,3\}$, be a $0-1$ matrix such that

$$
M=\left(\begin{array}{llll}
1 & 1 & 0 & 0 \\
0 & 1 & 1 & 0 \\
0 & 0 & 1 & 1 \\
1 & 0 & 0 & 1
\end{array}\right) .
$$

and $\left(\mathrm{X}_{M} \cdot \sigma_{M}\right)$ a Markov subshift (topological Markov chain) for the structure matrix $M$. Define a coding function $\Psi_{0}$ and a isomorphism $\Psi$ on $X_{M}$ by

$$
\Psi_{0}\left(\varepsilon_{1} \cdot \varepsilon_{2}\right)=\delta_{1}=\left\{\begin{aligned}
0 & \text { for } \varepsilon_{1}-\varepsilon_{2}=0 \\
1 & \text { for } \varepsilon_{1}=0 \\
-1 & \text { for } \varepsilon_{1}=1 \\
-1 & \text { for } \varepsilon_{1}=2 \\
1 & \text { for } \varepsilon_{1}=3
\end{aligned}\right\} \text { and } \varepsilon_{1}-\varepsilon_{2} \neq 0
$$

and for each $\omega \in X_{M}$ 


$$
\Psi(\omega)=\left\{\Psi_{0}\left(\sigma_{M}^{n-1} \omega\right)\right\}_{n=1}^{\infty} .
$$

Then we obtain.

\section{Proposition(3.1)}

Let $w$ be a set of the revolving sequences. Then the map $\Psi$ is one-one onto from $X_{M} \backslash\left(\varepsilon_{1}, \varepsilon_{2}, \ldots\right): \varepsilon_{j}=a$ for all $j$ and $a \in\{0,1.2,3\}\}$ to $W \backslash\{(0,0, \ldots)\}$. and satisfies a commutative relation

$$
\sigma \cdot \Psi=\Psi \cdot \sigma_{M} .
$$

Now denote a set of all finite revolving sequences with length $n$ by $w^{(n)}$ and the decomposition of $w^{(n)}$ by

$$
\begin{aligned}
w_{\varepsilon}(n)=\left\{\left\{\Psi_{0}\left(\varepsilon_{j} \cdot \varepsilon_{j+1}\right)\right\}\right. & n=1: \varepsilon_{1}=\varepsilon_{j=1} \text { and } \\
& \left.\left(\varepsilon_{1}, \ldots, \varepsilon_{n+1}\right) \text { is M-admissible }\right\} .
\end{aligned}
$$

and

$$
w_{(\varepsilon . \delta)}^{(n)}=\left\{\left(\delta_{1}, \ldots \delta_{n}\right) \in w_{\varepsilon}(n) ; \delta_{1}=\delta\right\} .
$$

Then we obtain.

\section{Proposition(3.2)}

(1) $w^{(n)}=U_{\varepsilon \in\{0,1,2,3\}^{W}}{ }^{(n)}$.

(2) $\left.w_{\varepsilon}^{(n)}=w_{(\varepsilon, 0)}^{(n)} \cup w_{(\varepsilon, 1-i)}^{(n)}\right)^{(n)}$,

(3) $\quad \sigma w_{(\varepsilon, 0)}^{(n)}=w_{\varepsilon}^{(n-1)}$ and

$$
\left.\sigma w_{(\varepsilon \cdot(-1)}^{(n)} \varepsilon\right)=w_{\varepsilon+1(\bmod 4)}^{(n-1)}
$$

(4) $\quad(-i) w_{\varepsilon}^{(n)}=w_{\varepsilon+1(\bmod 4)}^{(n)}$,

$$
(-i) w_{(\varepsilon .0)}^{(n)}=w_{(\varepsilon+1(\bmod 4) .0)}^{(n)} \text {, }
$$


and

$$
\left.(-i) w_{(\varepsilon \cdot(-i)}^{(n)}\right)^{(\varepsilon)}=w_{\left(\varepsilon+1(\bmod 4),(-i)^{\varepsilon+1}\right)}^{(n)} .
$$

Let $\ell$ be a map from $w^{(n)}$ to a line segment such that

$\ell\left(\delta_{1}, \ldots \delta_{n}\right)=$ segment which comnects $p\left(\delta_{1} \ldots \delta_{n}, 0\right)$ and $P\left(\delta_{1} \ldots, \delta_{n}, \delta_{n+1} \neq 0\right)$. where

$$
p\left(\delta_{1} \ldots \ldots \delta_{n}\right)=\sum_{k=1}^{n} \delta_{k}(1+i)^{-k} \text {. }
$$

By the way, define a n-step twindragon curve $D_{n}$ and a $n$ step dragon (paper folding) curve $H_{n}(F i g .3(a)(b)$ ) by
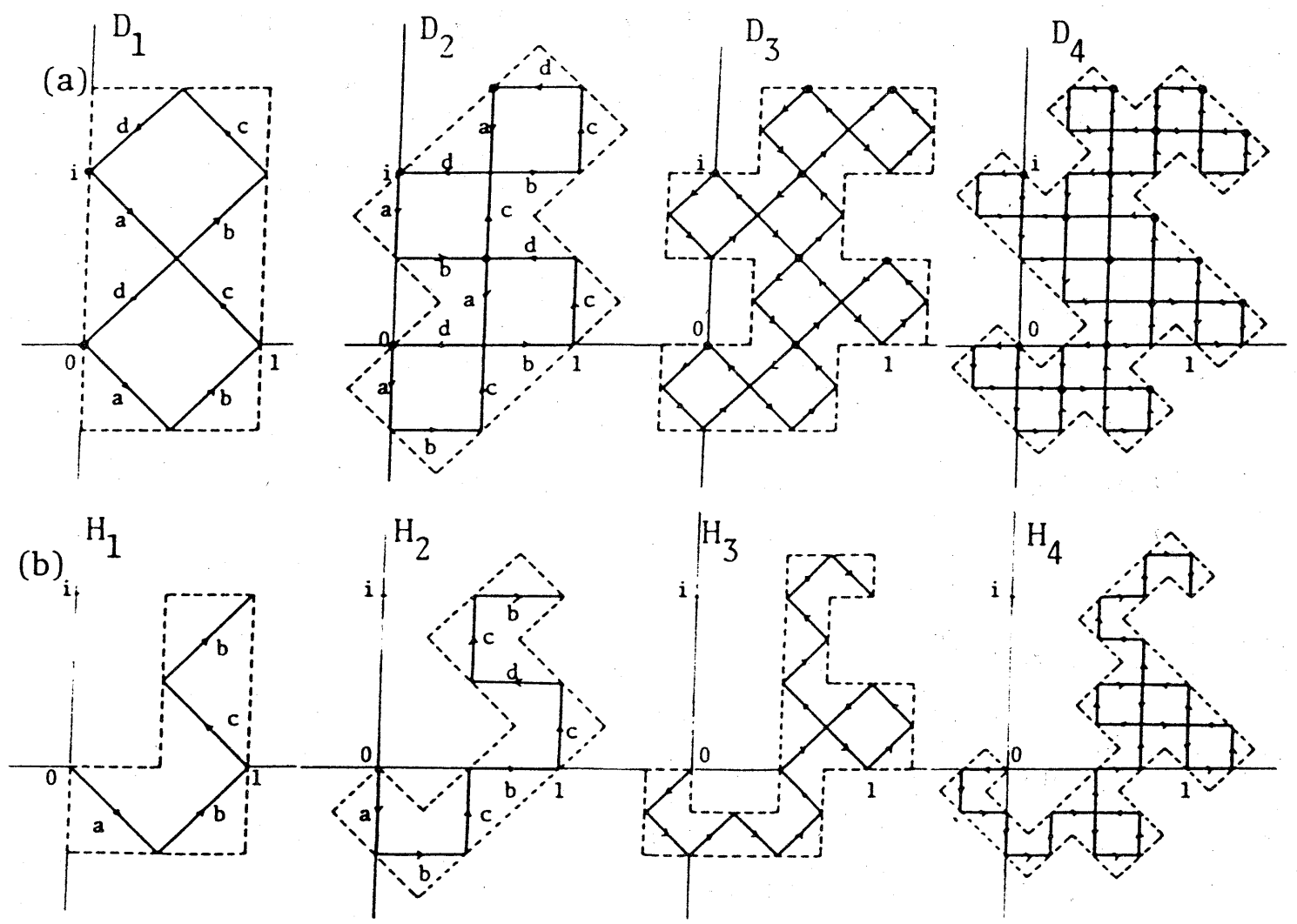

Fig. 3: Twindragon $\mathrm{D}_{\mathrm{n}}$ and Dragon $\mathrm{H}_{\mathrm{n}}$ and their boundaries. 


$$
\left.D_{n}=(1+i)^{-n_{K}\left[\theta_{T}\right.}{ }^{n}(a b c d)\right]
$$

and

$$
H_{n}=(1+i)^{-n_{K}}\left[\theta_{T}^{n}(a b)\right] .
$$

where $G=\{a b c d\}$ and $a$ homeomorphism $f$ is such that

$$
f(a)=1=-f(c) \text { and } f(b)=i=-f(d) \text {. }
$$

and an endomorphism $\theta_{\mathrm{T}}$ is defined by

$$
\theta_{T}: a \rightarrow a b, b \rightarrow c b, c \rightarrow c d, d \rightarrow a d .
$$

we notice the twindragon curve is tiled by two dragon curves. that is,

$$
D_{n}=H_{n} \cup\left(-H_{n}+1+i\right) .
$$

\section{Lemma (3.1)}

Let $\ell_{\varepsilon}^{(n)}$ and $\ell_{(\varepsilon, \delta)}^{(n)}$ be defined by

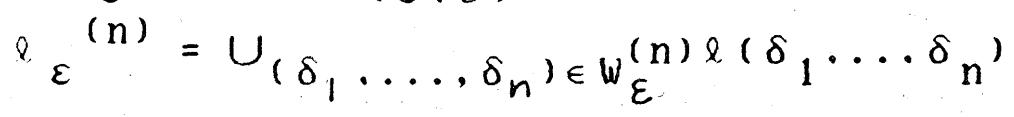

and

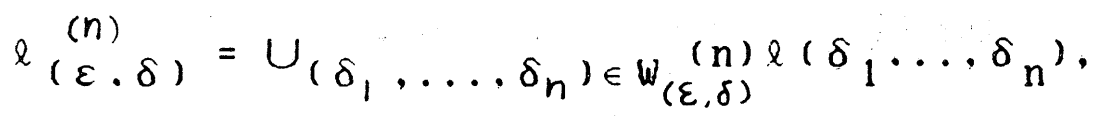

then $\ell_{(\varepsilon, \delta)}^{(n)}$ and $\ell_{\varepsilon}(n)$ are similar to the $(n-2)$-step and $(n-1)$-step dragon curve respectively (Fig. $4(a)(b))$.

Let $U$ be a closed square in section 2 , $U^{\prime}$ a closed square such that $U^{\prime}=U+1 / 2$ and $B^{\prime}{ }_{n+1}$ defined by

$$
B^{\prime}{ }_{n+1}=\partial\left(U_{x \in P_{n+1}} \times\left(\varepsilon_{0}, \ldots, \varepsilon_{n-1}\right)+(1+1)^{-(n-1)} U\right) .
$$

then

\section{Lemma (3.2)}

(1) The n-step twindragon curve $D_{n}$ is covered by a closed curve- ${ }^{\prime}{ }_{n+1}$ as an envelope (Fig.3ia)), that is.

$$
d_{0}\left(D_{n} \cdot B^{\prime}{ }_{n+1}\right)=\sup _{x \in B_{n+1}^{\prime}} \quad \inf _{y \in D_{n}}|x-y|=\left(\frac{1}{\sqrt{2}}\right)^{n+1} .
$$



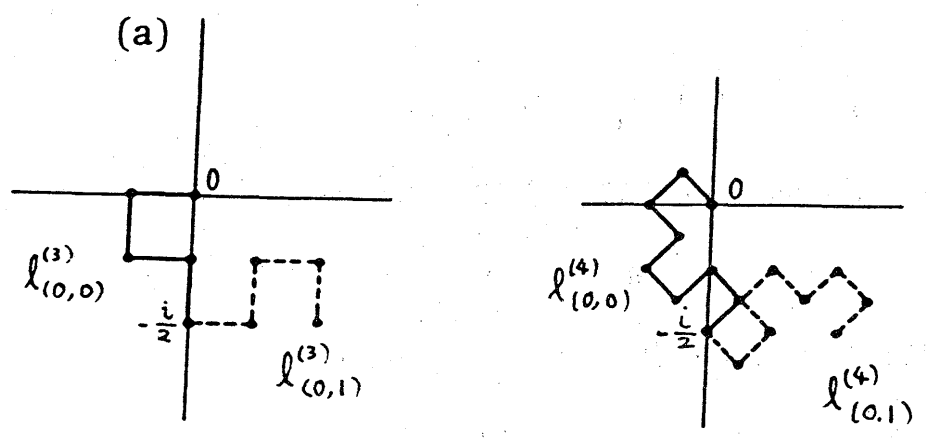

(b)

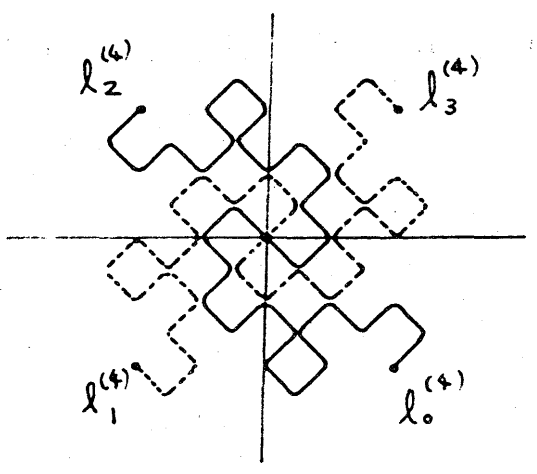

Fig.4: (a) Line segments $\ell_{(0,0)}^{(n)}$ and $\ell_{(0,1)}^{(n)}$ for $n=3,4$.

(b) Line segments $\left\{l_{\varepsilon}^{(n)}\right\}$ for $n=4$.

(2) The limit set $D_{T}$ of $\left\{D_{n}\right\}_{n=1}$ has a dragon boundary as its boundary.

Moreover using above lemma we can prove that

\section{Lemma (3.3)}

Let $H_{T}$ be the limit set of the paper folding curve $H_{n}$. Then the boundary of $H_{T}$ consists of three parts of the dragon boundary. Therefore $\operatorname{dim}_{H} \partial H_{T}=d i m_{H} \partial D_{T}=2 \log \beta_{0} / \log 2$. 
157

Put

$$
\begin{aligned}
& x_{(\varepsilon, \delta)}^{(n)}=\left\{\sum_{k=1}^{n} \delta_{k}(1+i)^{-k} ;\left(\delta_{1} \ldots \delta_{n}\right) \in W_{(\varepsilon, \delta)}^{(n)}\right\} \\
& x_{\varepsilon}^{(n)}=\left\{\sum_{k=1}^{n} \delta_{k}(1+i)^{-k}:\left(\delta_{1}, \ldots \delta_{n}\right) \in W_{\varepsilon}^{(n)}\right\},
\end{aligned}
$$

and let $X_{(\varepsilon, \delta)}$ and $X_{\varepsilon}$ be limit sets of $X_{(\varepsilon, \delta)}^{(n)}$ and $X_{\varepsilon}{ }^{(n)}$ respectively (Fig.5). Thus we can prove that

Lemma (3.4)

(1) $\quad(1+i) X_{(\varepsilon, 0)}=X_{\varepsilon}$

(2) $(1+i) X_{\left.(\varepsilon, 1-i)^{\varepsilon}\right)}=X_{\varepsilon+1(\bmod 4)}+(-i)^{\varepsilon}$.

(3) $\quad$ int $\left(X_{(\varepsilon, \delta)}\right) \cap$ int $\left.\left(X_{(\varepsilon,}, \delta,\right)\right)=\phi$ for

$(\varepsilon . \delta) \neq\left(\varepsilon^{\prime}, \delta^{\prime}\right)$,

and $\partial X_{(\varepsilon, \delta)^{\prime}} \cap \partial X_{\left(\varepsilon^{\prime}, \delta^{\prime}\right)}$ consists of parts of the dragon boundary.

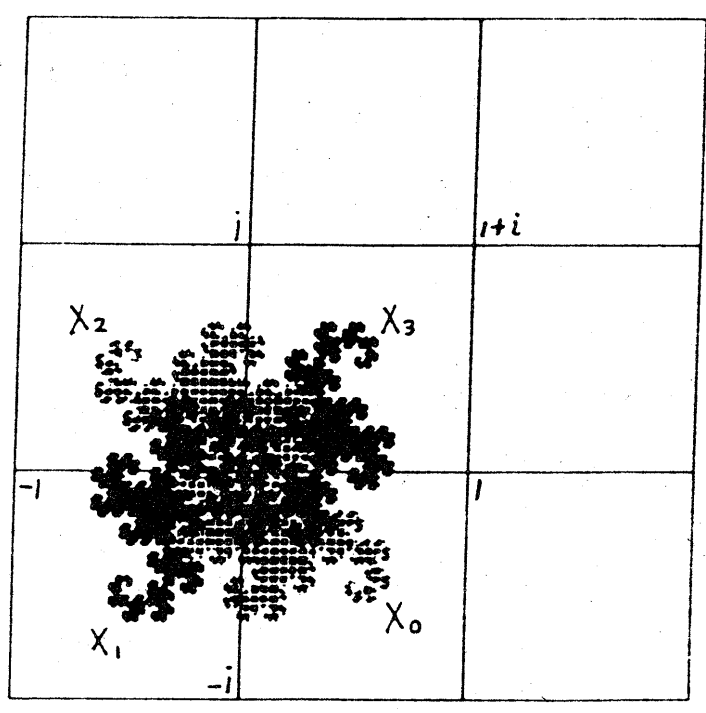

Fig. 5: Tetradragon X.

14 
Then putting

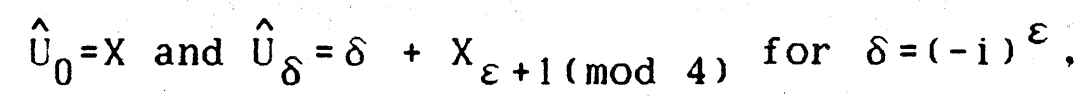

and let a map $T$ on $X$ be

$$
\mathrm{T} z=(1+i) z-[z]_{D}
$$

where ${ }^{[z]} D=\delta$ if $w \in \hat{U}_{\delta}$ for $\delta \in\{0,1,-i,-1 . i\}$,

then a transformation $(X, T)$ is well defined and induces the revolving expansion.

\section{Theorem(3.1)}

(1) The Lebesgue measure $\nu$ is invariant with respect to $(\mathrm{X} . \mathrm{T})$.

(2) the dynamical system $(X, T, \nu)$ is isomorphic to $\left(X_{M} \cdot \sigma_{M} \cdot \mu_{M}\right)$. where $\mu_{M}$ is a stationary Markov measure such that

$$
P=\left(\begin{array}{cccc}
1 / 2 & 1 / 2 & 0 & 0 \\
0 & 1 / 2 & 1 / 2 & 0 \\
0 & 0 & 1 / 2 & 1 / 2 \\
1 / 2 & 0 & 0 & 1 / 2
\end{array}\right) \quad \Pi=(1 / 4.1 / 4,1 / 4.1 / 4)
$$

\section{Remark 1:}

The dual algorithm of $(X, T, \nu)$ is constructed by taking a complex conjugate. $X^{*}=\bar{X}$, and putting

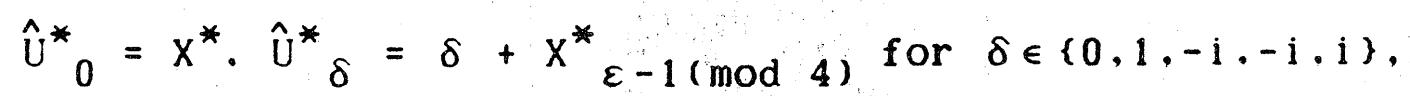

and

$$
T^{*} z=(1-i) z-[(1-i) z] D^{*}
$$

where $[w] D^{*}=\delta$ if $w \in \hat{U}^{*} \delta$. Then a dynamical system $\left(X^{*}, T^{*}, \nu\right)$ is the dual system for the system $(X, T, \nu)$ and 
induces the "converse" revolving expansion,

$$
z=\sum_{k=1}^{\infty} \delta_{k}^{*}(1-i)^{-k} \text {. }
$$

\section{Remark 2:}

If we choose formally the dual domain $x^{\#}$ as

$$
x^{\#}=U_{\varepsilon} x_{\varepsilon}^{\#} \text {, }
$$

where

$$
\left.x_{\varepsilon}^{\#}=\left\{\sum_{k=1}^{\infty} \delta_{k}^{*} k^{(1+i}\right)^{-k} ;\left(\delta_{1}^{*}, \delta_{2}^{*}, \ldots\right) \in w_{\varepsilon}^{*}\right\} .
$$

Then we obtain an interesting picture (Fig.6). This selfsimilar fractal curve is already studied by $P$. Lévy in $1938^{8)}$.

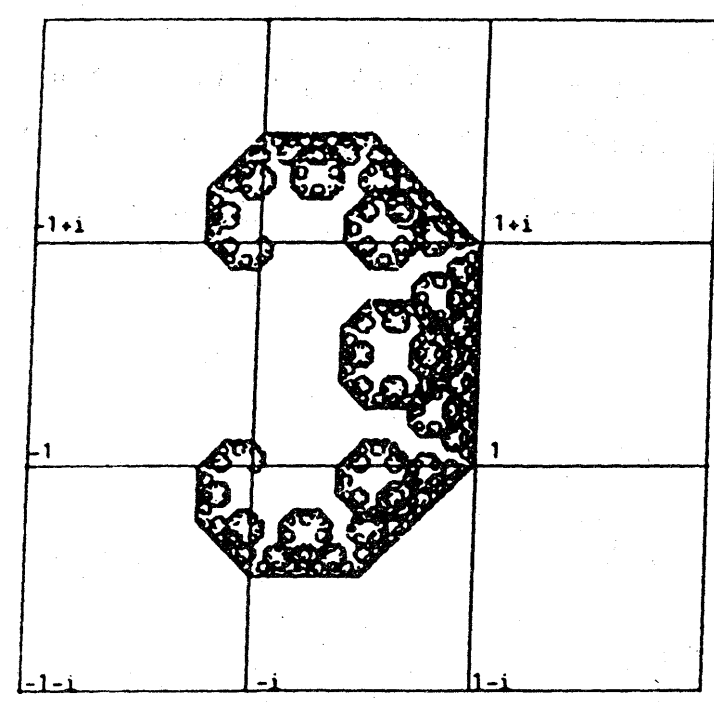

Fig. 6: $\mathrm{X}_{0}^{\#}$. 
4. DUAL SYSTEM ON CROSS DRAGON

Let $E=\left(E_{j, k}\right), 1 \leqq j, k \leqq 4$, be a matrix such that

$$
E=\left(\begin{array}{llll}
0 & 1 & 1 & 0 \\
0 & 0 & 1 & 1 \\
1 & 0 & 0 & 1 \\
1 & 1 & 0 & 0
\end{array}\right)
$$

We consider $E$ as the structure matrix for states $\Gamma=$ $\{0, \mathrm{i},-1+\mathrm{i},-1\}$ by a correspondence $\tau:\{1,2,3,4\} \rightarrow \Gamma$ such that $\tau[1]=0, \quad \tau[2]=i, \quad \tau[3]=-1+i$ and $\tau[4]=-1$, that is, let $V$ be a set of infinite sequences generated by the structure matrix $E$,

$$
v=\left\{\left(r_{1}, 2, \ldots\right) ; E_{\gamma_{j}}, \gamma_{j+1}=1, r_{j} \in \Gamma \text { for all } j \in N\right\}
$$

and $\sigma$ a shift on $V$. Then the system $(V, \sigma)$ is a Markov subshift. Let $v^{(n)}$ be a set of E-admissible sequences with length $n$ and $v_{\gamma}^{(n)}$ be.

$$
v_{r}^{(n)}=\left\{\left(r_{1}, \ldots r_{n}\right) \in v^{(n)} ; r_{1}=r\right\} \text {. }
$$

Notice that nonzero entries of the structure matrix can be uiritten as $E_{\tau[k], \tau[(k+1) \bmod 4]}=E_{\tau[k], \tau[(k+2) \bmod 4]}$ and denote these two admissible states after $r=\tau[k]$ by $r[1]=$ $\tau[(k+1) \bmod 4]$ and $\gamma[2]=\tau[(k+2) \bmod 4]$ respectively.

\section{Property(4.1)}

(1) $V^{(n)}=U_{\gamma \in\{0, i,-1+i,-1\}^{V}} r^{(n)}$,

(2) $\sigma v_{\gamma}^{(n)}=v_{\gamma[1]}^{(n-1)} \cup v_{\gamma[2]}^{(n-1)}$,

(3) $\quad i V_{r}^{(n)}+i=v_{r[1]}^{(n)}$

and

$$
-v_{r}^{(n)}+(-1+i)=v_{\gamma[2]}^{(n)} .
$$


We realize a sequence $\left(r_{1}, \ldots, r_{n}\right)$ to a point $\mathrm{p}\left(r_{1}, \ldots, r_{\mathrm{n}}\right)$ by

$$
p\left(r_{1}, \ldots, r_{n}\right)=\sum_{k=1}^{n} r_{k}(1+i)^{-k} \text {. }
$$

According to the set of sequence $V^{(n)}$ and $V_{\gamma}^{(n)}$ let $Y^{(n)}$ and $Y_{r}{ }^{(n)}$ be sets of points $\left\{p\left(r_{1}, \ldots, r_{n}\right)\right\}$.

It is verified that $d\left(Y^{(n)}, Y^{(n+1)}\right) \leqq\left(\frac{1}{\sqrt{2}}\right)^{n}$.

in the Hausdorff metric. So $Y^{(n)}$ and $Y_{r}{ }^{(n)}$ converge to $Y$ and $Y_{r}$ respectively as $n \rightarrow \infty$ (Fig.7).

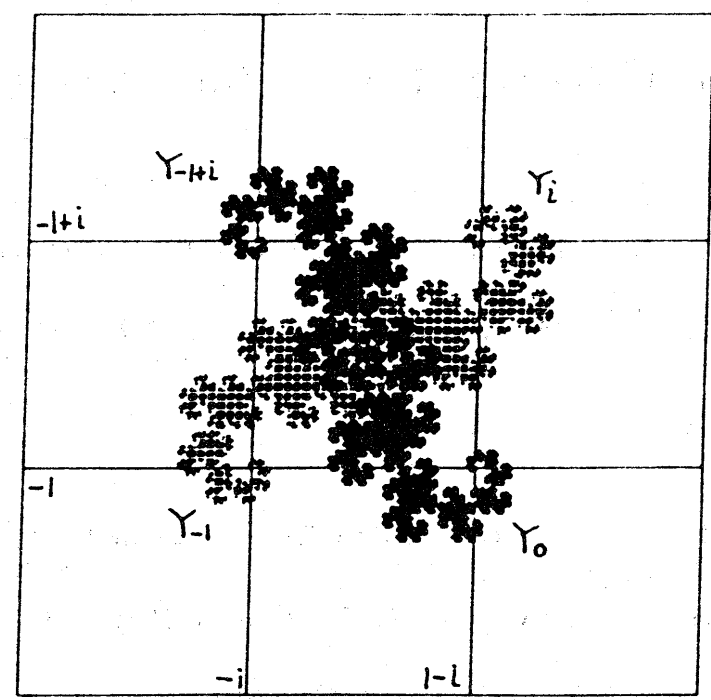

Fig.7: Cross dragon Y. 


\section{$\operatorname{Lemma}(4.1)$}

Let $Y=\left\{\sum_{k=1}^{\infty} r_{k}(1+i)^{-k}:\left(r_{1}, r_{2}, \ldots\right) \in V\right\}$ and $Y_{r}=$ $\left\{\sum_{k=1}^{\infty} r_{k}(1+i)^{-k}:\left(r_{1} \cdot r_{2} \cdots\right) \in V r\right\}$. Then the sets $Y$ and $Y_{\gamma} \cdot \gamma \in \Gamma$. satisfy following properties.

(1) $Y=U_{\gamma} \in\{0, \mathrm{i},-1+\mathrm{i},-1\}^{Y} \gamma$

(2) $(1+i) Y_{r}-\gamma=Y_{r[1]} \cup Y_{r[2]}$,

(3) $\quad$ iY $r+1=Y_{r}[1]$

and

$$
-Y_{r}+1+i=Y_{\gamma[2]} \cdot
$$

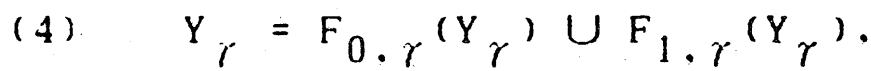

where $F_{0, \gamma}$ and $F_{1, \gamma}$ are contraction maps such that

$$
F_{0 \cdot r}(z)=(1+i)^{-1}(i z+r+1)
$$

and

$$
\Gamma_{1, \gamma}(z)=(1+i)^{-1}(-z+r+1+i) \quad \text { for each } r \in \Gamma \text {. }
$$

Recall another approach for selfsimilar fractal sets proposed by Hutchinson ${ }^{7}$ ) using a set of contraction maps.

Theorem (Hutchinson ${ }^{7}$ ),

Let $\mathscr{L}$ be a finite set of contraction maps $\left\{S_{1} \ldots, S_{M}\right\}$ on a metric space. Then there exists a unique closed bounded set $K$ such that $K=\bigcup_{j=1}^{M} S_{j}(K)$. Moreover. let $\mathscr{L}(A)=\cup_{j=1}^{M} S_{j}(A)$ and $\mathscr{L}^{\mathrm{P}}(A)=\mathscr{L}\left(\mathcal{L}^{\mathrm{P}-1}(A)\right)$ for arbitrary set $A$, then $\mathcal{L}^{\mathrm{P}}(\mathrm{A}) \rightarrow \mathrm{K}$ in the Hausdorf $\mathrm{f}$ metric as $\mathrm{p} \rightarrow \infty$ for closed bounded set $A$. 
Thus we can say that the limit sets $\left\{Y_{\gamma}\right\}$ are invariant sets for the contraction maps $\left\{F_{0}, \gamma, F_{1}, \gamma\right\}$. Notice that the set $\left\{X_{\varepsilon}^{*}\right\}$ in section 3 are the invariant set for the contraction maps $\left\{G_{0, \varepsilon}^{*}, G_{1, \varepsilon}^{*}\right\}$ for each $\varepsilon \in\{0,1,2,3\}$, where

$$
G_{0, \varepsilon}^{*}(z)=(1-i)^{-1} z \text { and } G_{1, \varepsilon}^{*}(z)=(1-i)^{-1}\left(i z+i{ }^{\varepsilon}\right),
$$

that is,

$$
X_{\varepsilon}^{*}=G_{0, \varepsilon}^{*}\left(X_{\varepsilon}^{*}\right) \cup G_{1, \varepsilon}^{*}\left(X_{\varepsilon}^{*}\right)
$$

and for $\mathscr{L}=\left\{G_{0, \varepsilon}^{*} \cdot G^{*}{ }_{1, \varepsilon}\right\}$

$$
G_{0 . \varepsilon}^{*}\left(\mathcal{L}^{n}(0)\right)=X_{(\varepsilon, 0)}^{*}
$$

and

$$
G_{1, \varepsilon}^{*}\left(\mathcal{L}^{n}(0)\right)=X_{\left(\varepsilon, i^{\varepsilon}\right)}^{*(n+1)} \text {. }
$$

Then we obtain

\section{Theorem(4.1)}

Let $\left(Y_{\gamma}\right.$ ) satisfy $Y_{\gamma}=F_{0, \gamma}\left(Y_{\gamma}\right) \cup F_{1}, \gamma\left(Y_{\gamma}\right)$ for each $r \in \Gamma$. and $Y=U_{\gamma \in\{0, i,-1+i,-1\}}{ }^{Y}$. Then

(1) Each set $Y_{r}$ is a dragon with end points 0 for $Y_{-1}, 1$

for $Y_{0} \cdot 1+i$ for $Y_{i} \cdot i$ for $Y_{-1+i}$ and $(1+i) / 2$ in common.

(2) The set $Y$ is tiled by four dragons $\left\{Y_{\gamma}\right\}$, that is.

and

$$
Y=U_{\gamma \in\{0, i,-1+i,-1\}^{Y}}
$$

$$
\lambda\left(Y_{r} \cap Y_{r}\right)=0 \quad \text { for } r \neq r^{\prime} \text {. }
$$

We call the set $Y$ a cross dragon. 
Let a map $S$ on $Y$ be

$\mathrm{Sz}=(1+\mathrm{i}) \mathrm{z}-[(1+\mathrm{i}) \mathrm{z}]_{\mathrm{C}}$.

where ${ }^{[w]}{ }_{C}=\gamma$ if $w \in \gamma+\left(Y_{\gamma[1]} \cup Y Y_{r[2]}\right)$. Then (Y.S) is well defined and induces an expansion

$$
z=\sum_{k=1}^{\infty} r_{k}(1+i)^{-k} \quad \text { for a.e. } z \in Y \text {. }
$$

Now let $Y^{*}=\{x+i y: 0 \leqq x, y<1\}$ and a map $S^{*}$ be

$$
S^{*} z=(1+i) z-[(1+i) z] \text {, }
$$

where $[w]=[\operatorname{Re}(w)]+i[\operatorname{Im}(w)]$ for $z \in \mathbb{C}$. This system is

equivalent to a group endomorphism $T_{L}$ on the torus $T^{2}$ such that

$$
T_{L}\left(\begin{array}{l}
x \\
y
\end{array}\right)=\left(\begin{array}{rr}
1 & -1 \\
1 & 1
\end{array}\right)\left(\begin{array}{l}
x \\
y
\end{array}\right)-\left(\begin{array}{l}
{[x-y]} \\
{[x+y]}
\end{array}\right) .
$$

Theorem(4.2)

(1) The Lebesgue measure $\lambda$ is invariant with respect to $(Y, S)$

(2) The cross dragon system $(Y, S, \lambda)$ is actually the dual system for $\left(Y^{*} \cdot S^{*}, \lambda\right)$.

\section{Remark:}

The cross dragon system $(Y, S, \lambda)$ is isomorphic to a following map on the torus.

$$
T^{\dagger}\left(\begin{array}{l}
x \\
y
\end{array}\right)=\left(\begin{array}{rr}
1 & 1 \\
-1 & 1
\end{array}\right)\left(\begin{array}{l}
x \\
y
\end{array}\right)+\left(\begin{array}{r}
-1 \\
1
\end{array}\right)-\left(\begin{array}{l}
{[x+y-1]} \\
{[-x+y+1]}
\end{array}\right) \text {. }
$$




\section{REFERENCES}

1) D. Knuth, The arts of computer programming II, section 4.1, Addsion Wesley, 1969.

2) C. Davis and D. Knuth, Number representation and Dragon curves I. J.of Recreational Math. 3 (1970)66-81.

3) M. Mizutani and Sh. Ito, Dynamical systems on Dragon domains. to appear.

4) M. Mizutani and Sh. Ito. A New Characterization of Dragon and Dynamical system, to appear.

5) F.M. Dekking, Recurrent Sets, Advance Wath.44(1982)78-104.

6) F.M. Dekking. Replicating Super figures and Endomorphisms of Free groups, J. Combi. Theor.A32(1982)315-320.

7) J.E. Hutchinson, Fractals and Selfsimilarity, Indiana Univ. Math. J. $30(1981) 713-747$.

$8)$ P. Lévy, Les courbes planes ou gauches et les surfaces composées de parties semblables au tout, J.de l'Ecole Polytechnique Serie III7-8(1938)227-291. 